\title{
Statistical study of neutron star glitches
}

\author{
Eya, Innocent Okwudili, Urama, Johnson O.
}

Department of Physics and Astronomy, University of Nigeria, Nsukka, Enugu State, Nigeria

\section{Email address:}

innocent.eya@unn.edu.ng (Eya, I. O.), innoson02@yahoo.com (Eya, I. O.), johnsonurama@yahoo.com (Urama, J. O.)

\section{To cite this article:}

Eya, Innocent Okwudili, Urama, Johnson O.. Statistical Study of Neutron Star Glitches. International Journal of Astrophysics and Space Science. Vol. 2, No. 2, 2014, pp. 16-21. doi: 10.11648/j.ijass.20140202.11

\begin{abstract}
Neutron star glitches; spanning a period of 42 years of pulsar timing were studied. These glitches are from Radio, X-ray, Anomalous X-ray and Milliseconds Pulsars. Radio Pulsars dominates the glitch events, contributing $87 \%$ of the glitches. Pulsars of characteristic age bracket $10^{3}$ to $10^{5}$ yrs dominated the glitch events, at a rate of 5.2 glitches per year per pulsar. Pulsar of the above age bracket exhibits large size glitches compared to others. A large frequency spin-up $(\triangle v)$ is generally associated with large frequency derivative jump $(\triangle \dot{v})$. The distribution of the glitch magnitude $(\triangle v / v)$ is bimodal reaffirming dual glitch mechanism, but that of spin-up $(\triangle v)$ is tending towards multi-modal. Moreover, glitches in Vela pulsar and PSR J0537-6910 showed strong elasticity of the objects, suggesting that the interiors of these objects are in thermal equilibrium. Glitches from PSR J1740-3015 and PSR J1341-6220 appeared to occur in groups, suggesting that their interior fluid is switching between two phases. We discussed the glitch activity of young pulsars in terms of vortex creep model.
\end{abstract}

Keywords: Star, Neutron, Pulsar, Glitch, General

\section{Introduction}

Neutron stars are extremely dense, degenerate stellar corpses composed mostly of densely packed neutrons [1]. They are one of the products of supernovae event, which mark the death of middle mass stars. These stellar corpses have mass of about 1.4 solar masses and radius of $10 \mathrm{Km}$. Neutron star manifests itself as pulsars [2] such as: Radio, X-ray, Anomalous X-ray (AXP), Millisecond pulsars, etc. In this study, we laid emphasis in the above-mentioned manifest (class).

The rotation rate of pulsars decreases monotonically as kinetic energy is lost via the emission of electromagnetic radiations and particles [3]. Occasionally, the pulsar is spun-up in an event known as 'glitch'. A glitch is sudden increase in pulse frequency $\boldsymbol{v}$ of otherwise decelerating pulsar. This event is often followed; by an exponential relaxation toward the pre-glitch frequency [4]. The fractional increases in pulse frequency (known as glitch magnitudes) are in the range of $\Delta v / \nu \sim 10^{-11}-10^{-5}$, while the fractional changes in spin-down rate due to glitches (where they are resolvable) are in the range of $\Delta \dot{v} / \dot{v} \sim 10^{-1}-10^{-4}[5 ; 6]$. However, observational selection limits the low end of this distribution. On the other hand, glitches are believed to be as a result of starquake [e.g.,7; 8;9] or by abrupt transfer of angular momentum from a faster rotating superfluid interior to the observable crust [e.g.10;11;12]. Pulsar glitches is of utmost important to astrophysics as this is the only physical process that originates from pulsar's interior; study of such glitches is an insight on the state of matter in the neutron star interior which involves the physics of extreme density.

Meanwhile, in this study, 315 glitches in 102 neutron stars from the ATNF Pulsar Glitch database (http://www.jb. man.ac.Uk/pulsar/glitches.html) as compiled by [6] were analyzed. The data spans $42 y$ rs of pulsar timing. Out of the 315 glitches, Radio pulsar contributed 274 glitches, X-Ray pulsar 26, AXPs 14, and Milliseconds pulsar 1. Also out of the 102 glitchers, 54 have glitched once. Pulsars that glitched more than once, but less than six times are 37 in number. While those that glitched at least six times are just 11. Out of these 11 pulsars which was used in comparing the glitches from various classes of neutron star, AXP and $\mathrm{X}$-ray pulsar contributed one each while Radio pulsars have nine and Milliseconds pulsar none.

\section{Glitch Rate and Neutron Star Characteristic Age}

Pulsars of characteristic age $\left(\tau_{c}=-v / 2 \dot{v}\right) 10^{3} \mathrm{yr}$ to $10^{5} \mathrm{yr}$ exhibit a high glitch rate. Pulsars in this age bracket contributed 196 glitches at rate of 5.2 glitches per pulsar reaffirming the suggestion of [13] that pulsars of this age bracket are good targets for glitch monitoring project. The 
eleven pulsars with at least six glitches belong to this group. Pulsars of age bracket $\left(10^{5}-10^{7}\right)$ years contributed 112 glitches with a lower rate of 1.9 glitches per pulsar. The summary of this analysis is as shown in Table 1 and in Figure 1.

Table 1. Glitch rate in different age bracket.

\begin{tabular}{|c|c|c|c|c|c|c|c|c|}
\hline Age (years) & $\begin{array}{l}\text { Number of } \\
\text { Pulsars }\end{array}$ & $\begin{array}{l}\text { Number of } \\
\text { Glitches }\end{array}$ & $\begin{array}{c}\text { Number of } \\
\text { Glitch per } \\
\text { Pulsar } \\
\end{array}$ & $\begin{array}{c}\text { Percentage of } \\
\text { Glitch in each age } \\
\text { group } \\
\end{array}$ & $\begin{array}{c}\text { Number of } \\
\text { Pulsar in Last } \\
\text { Decade } \\
\end{array}$ & $\begin{array}{c}\text { Number of } \\
\text { Glitches in } \\
\text { Last Decade } \\
\end{array}$ & $\begin{array}{c}\text { Number of Glitch } \\
\text { Per Pulsar in Last } \\
\text { Decade } \\
\end{array}$ & $\begin{array}{l}\text { Percentage of Glitch in } \\
\text { each age group in last } \\
\text { decade }\end{array}$ \\
\hline $10^{1}-10^{3}$ & 1 & 2 & 2.0 & 0.6 & 1 & 2 & 2.0 & 1.2 \\
\hline $10^{3}-10^{5}$ & 38 & 196 & 5.2 & 62.2 & 28 & 112 & 4.0 & 65.0 \\
\hline $10^{5}-10^{7}$ & 59 & 112 & 1.9 & 35.6 & 32 & 56 & 1.8 & 32.6 \\
\hline $10^{7}-10^{9}$ & 4 & 5 & 1.3 & 1.6 & 2 & 2 & 1.0 & 1.2 \\
\hline Sum & 102 & 315 & 10.4 & 100 & 63 & 172 & 8.8 & 100.0 \\
\hline
\end{tabular}
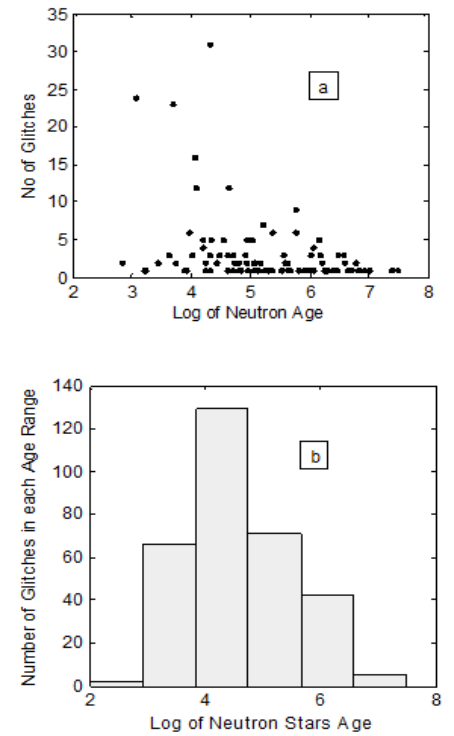

Figure 1. Distribution of glitches with pulsar characteristic age: (a) shows the number of glitch in a pulsar while (b) gives the average glitch per pulsar for different neutron star ages bracket.

\section{Glitch Sizes and Neutron Star Characteristic Age}

There is no any continuous linear relationship between the glitch sizes and pulsar ages; rather, AXPs glitches are always large. This features of AXPs that, [14] attributed to generic factor. However, within a class of neutron star, pulsars of characteristic age $\left(10^{4}-10^{5}\right)$ years tend to have larger glitch sizes than the very young $\left(<10^{4} \mathrm{yrs}\right)$ and old $\left(>10^{6} \mathrm{yrs}\right)$ pulsars. This result is in line with the idea of superfluid content of neutron star interior and temperature dependency of glitch mechanisms; as very young pulsars are too hot for vortices pinning and unpinning to work effectively and old pulsars are slow to accumulate large stress that results in a large glitch.

However, the distribution of glitch magnitude $\Delta v / v$ is bimodal Fig.2a (upper panel). This result reinforced the report of $[5 ; 6]$. The first modal-bin in this distribution contains 47 glitches centered at -8.56 corresponding to glitch magnitude of $2.75 \times 10^{-9}$. This is a typical Crab-like glitch though seven out of the 23 glitches in Crab Pulsar are greater than this value. The second modal-bin contains 32 glitches centered at -6.25 corresponding to glitch magnitude of 5.62 $\mathrm{X} 10^{-7}$. This is a typical PSR J0537-6910-like glitch, one magnitude less than Vela-like glitches. However, two of the Vela glitches are less than this magnitude, and one equals. The dip at -7.18 corresponding to glitch magnitude $6.61 \mathrm{X}$ $10^{-8}$ is not artificial. It strongly suggests dual glitch mechanisms with respect to glitch magnitude.

Moreover, the distribution of the spin-up ( $\Delta v)$ (Fig.2b lower panel) is not yet specific; more glitches are needed for full understanding of its evolution. Clearly, it is not uni-modal; there are demarcations among glitches of various sizes of which this paper strongly suggests that the spin-up distribution is tending towards multi-modal distribution.
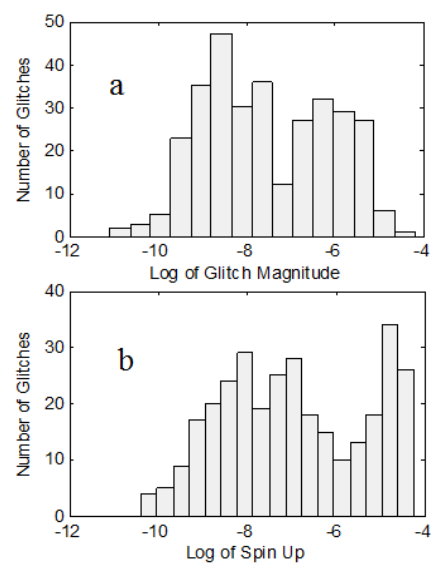

Figure 2. Histogram of the distributions of glitch sizes. (a) Glitch magnitude (b) Spin-up in pulse frequency.

\section{Glitch Magnitude $(\Delta v / v)$ and the Fractional Spin-down Rate $(\Delta \dot{v} / \dot{v})$}

We investigated the relationship between the glitch magnitude and the fractional spin-down rate. The value of correlation coefficient (0.16) obtained showed a weak correlation. This result is inevitable due to errors associated with the values of the fractional spin-down rate, or that different process regulates them. However, on plotting the $\log$ of these quantities, we obtain a correlation coefficient of 0.50 . This is an indication that the two quantities have a form of power relation (Fig.3b). 

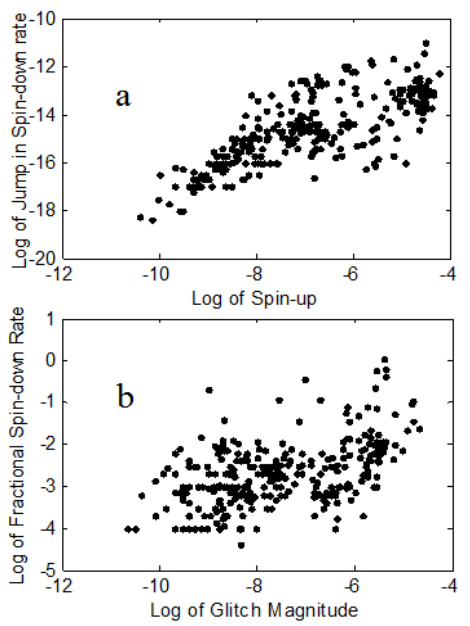

Figure 3. Scatter plot of glitch parameters; (a) Variation of spin-up with jump in spin-down rate (b) Variation of glitch magnitude with fractional spin-down rate.

Meanwhile, from the source of data it was reported that measurements of $\Delta \dot{v}$ are not extremely accurate, but for large glitches they are likely to be fairly well estimated. Most measurement of $\Delta \dot{v} / \dot{v}$ are positive as expected; if frequency recovery is understood as an exponential-like decrease to pre-glitch frequencies. However, a number of measurements are negative (54 in number). These negative measurements, [15] suggested being sharp features in timing noise mistaken to be glitches. In Fig.3a, the Spin-up $(\Delta v)$ is plotted against the corresponding jump in frequency derivative $(\Delta \dot{v})$. The plot includes 292 glitches that have finite, and non-zero frequency derivative jump. It can be seen from the figure that the two quantities correlate (having a correlation coefficient of 0.78 ), indicating that large frequency spin-ups $(\Delta v)$ are generally accompanied by large frequency derivative $(\Delta \dot{v})$ jumps. This result is an evident that magnetic field strength of the pulsar magnetosphere does not vary during glitch events.

\section{The Glitch Sizes and Time Interval between Glitches}

One expects that the glitch size should be a function of the elapsed time between glitches. On correlating the glitch size and elapsed time between glitches for an ensemble of pulsars, the result (correlation coefficient is 0.08) showed that there is no strong relationship between the glitch size and the elapsed time between glitch events for an ensemble of pulsars. However, on picking pulsars that have glitched at least six times and in analogy to Hooke's law of elasticity, Vela pulsar and PSR J0537-6910 showed a perfect linear relationships between the cumulative glitch magnitude ( $\Delta v / v)$ and time to the next glitch. Both have correlation coefficients of 1.00. In this analogy, the magnitude of stress built up in the crust due to pulsar spin-down which is a function of time is viewed as the force on an elastic material (in Hooke's law), while the size of glitch is viewed as elongation of the elastic material due to applied force. These results are as shown Figure 4. For the two pulsars with a perfect relationship between the glitch magnitude and elapsed time, the line is a least square fit obeying a simple equation that can be used to predict the magnitude of the next glitch as follows; for Velar pulsar; $\Delta v / v=1.95 \times 10^{-9}$ days $-4.3 \times 10^{-8}$, While for PSR J0537-6910 we have; $\Delta v / v=2.44 \times 10^{-9}$ days $-3.3 \times 10^{-7}$

However, cumulative glitch magnitude of the other pulsars could not fit perfectly with linear or other basic function. Glitches from PSR J1740-3015 and PSR J13416220 appear to occur in groups (large glitch separating small glitches at regular interval). This behavior is an indication that the interior of these pulsars are inhomogeneous and not in thermal equilibrium unlike Vela and PSR J0537- 6910. There can still be series of phase transition involving the matter within the contents of the star. The implication of this is that the mechanism producing glitches in this pulsar are of two or more types. The small size glitches from these pulsars could be a result of starquake, while the giant ones can be due to vortices unpinning especially the one, which resulted from the thermal driven mechanism of [16].

Finally, for that of Crab pulsar, the irregularity in its cumulative glitch magnitude coupled with the small size glitch it exhibit is a clear indication that starquake occurs in this pulsar at irregular intervals. The irregular starquake may be due to irregular variation of the pulsar's ambient magnetic field. In addition, its high spin-down rate may result in unevenly distribution of stress on the crust, which can lead to starquake of different magnitude at equal time interval.

\section{Cumulative Distribution of Glitch Sizes}

The cumulative distribution of glitch magnitude $(\Delta v / v)$ of pulsars with at least ten glitches revealed that the two pulsars that obeyed Hooke's law (i.e. Vela and PSR J05376910) have a specific glitch pattern. The distribution is continuous and regular (as shown in Fig.5). This result strongly suggests regularity of angular momentum impact on the crust of these pulsars during glitch. For other pulsars, the irregular pattern in the cumulative distributions of glitch magnitude is an indication that the glitch process in them is irregular, resulting in glitches of any size.

\section{Discussion}

\subsection{Number of Glitches per Pulsar}

The results present in this paper, suggests that how frequent a pulsar glitch depend largely on its characteristic age. This result is in agreement with most glitch models, from the fact that young pulsars do spin fast, there-by building-up stress in the crust more frequently than in older pulsars. Releasing of this stress leads to glitch.

In addition, a decade alone recorded more than $50 \%$ of 
the known glitches. This is inevitable due to increase observation of active pulsars and the use of more sensitive instruments than in past. Within this time scale, the youthful pulsars still dominate the glitch events. This result points towards field intensity of the pulsars magnetosphere playing a role in glitching processes. The spin-down rate which is a result of the interaction between the pulsars magnetosphere and the ambient magnetic field can also induce glitch more frequently in pulsars of high field intensity (corresponding to young pulsars and AXPs) than in those with low surface magnetic field intensity. This is because the electromagnetic breaking torque for high field intensity pulsars will be larger than in stars with low field intensity (very old pulsars). The effect of this is high rate of differential rotation in stars of high field intensity; leading to frequent stress build up (in the interface of superfluid interior and crust) that finally results in glitches in such stars. This process is a general phenomenon among all the manifestation of neutron stars and suggests that processes leading to glitch should be age dependent.

\subsection{Cumulative Glitch Magnitude and Time to the Next Glitch}

From the results obtained in section 5, it strongly supports the idea that stress is the actual cause of glitch in some pulsars. Those that can be fitted with Hooke's Law of elasticity, (Vela Pulsar and PSR J0537-6910) we suggest have a more homogeneous interior than others (Fig 4 and 5) do. This is because there is strong evidence of regular transfer of angular momentum within the star components for the glitch magnitude to follow a specific pattern with time.
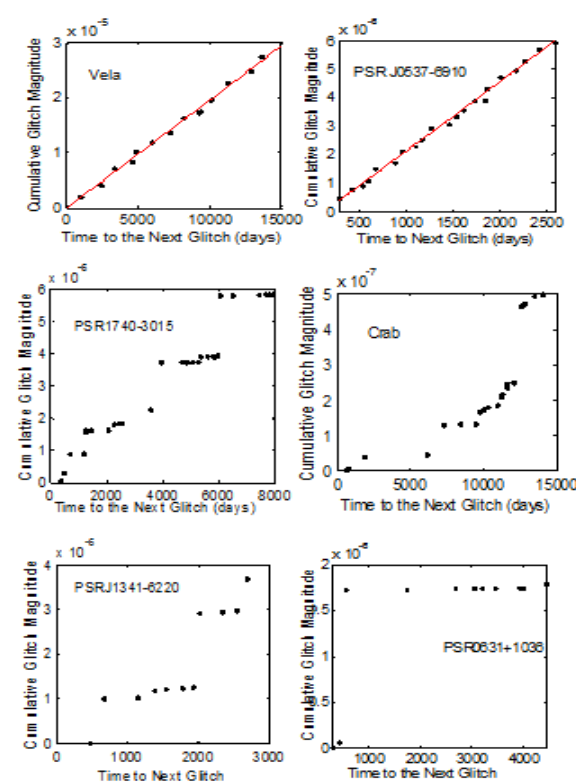

Figure 4. Cumulative glitch magnitude as a function cumulative glitch time for pulsars with many glitches.

In addition, it showed that the interiors of these stars are almost in thermal equilibrium with the inner crust. The temperature gradient between the layers of the star that triggers glitch is too low; this allows vortex unpinning and repining to be periodic. Another evidence for this is seen in the distribution of cumulative magnitude of these pulsars in section 6, where Vela and PSR J0537-6910 showed a continuous distribution and specific glitch pattern of three and two respectively, unlike other pulsar, which had neither continuous distribution nor specific glitch pattern.

\subsection{Radio Pulsar Glitches}

Glitch sizes in radio pulsars cover a wide range of $10^{-11}$ to $10^{-6}$, with the Crab-like glitches occupying the lower end while Vela-like glitches occupies the larger end. The starquake models can explain the glitches from Crab-like and other old radio pulsar. This is from the fact that the internal temperature of these very young pulsars are still too high for the glitch mechanism due to vortex pinning to work efficiently as it does for Vela-like pulsars.
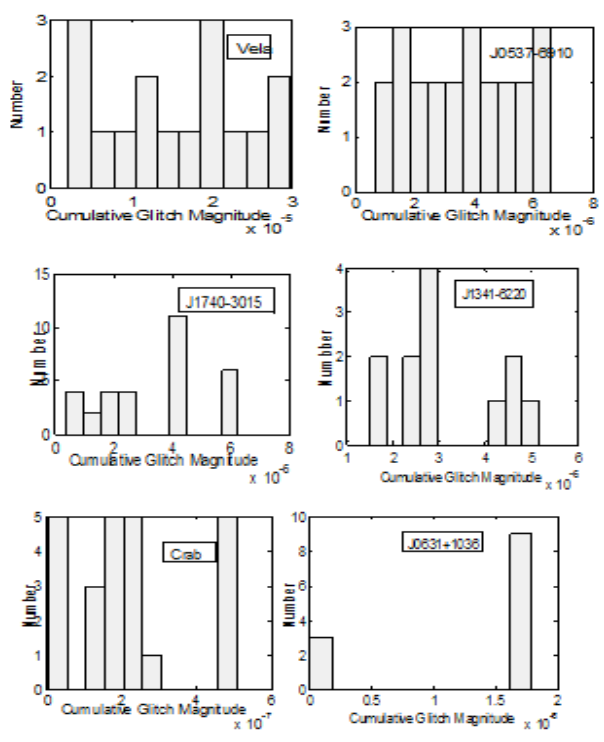

Figure 5. Cumulative distribution of glitch magnitude for pulsars with more than 10 glitches

On the other hand, for the old radio pulsars, the rotation lag between their components (i.e. superfluid and solid crust) are not large enough to produce large glitches while for Vela-like pulsars, the internal temperature is cooler and the rotation lag is much thereby building a large stress which will lead to large glitch whenever vortices are rearranged.

\subsection{AXPs Glitches}

Glitches in AXPs are among the largest ever seen in a neutron in star. They have magnitude $(\Delta v / v)$ up to $6.5 \mathrm{x}$ $10^{-5}$. Such a behavior may not be due to angular momentum transfer alone, but due to generic factor. Until there are a good number of glitches in AXPs, the actual mechanism lead to its glitches will still be elusive. Though [14] has suggested that the glitches in AXPs may be due to unpinning of vortices from nuclei in the lower crust. 


\subsection{X-Ray Pulsar Glitches}

For now, only PSR J0537-6910 is the X-Ray pulsar with significant number of glitches for statistical analysis. It is the most glitching pulsar of our time at a rate of 2.2 glitches per year. The glitch sizes are of the order of $10^{-7}$. Interestingly, there is a strong linear relationship between the cumulative glitch magnitude and the time to the next glitch (Fig.4) in this pulsar. The frequency of glitch in this pulsar keeps nobody in doubt that the most likely explanation for these glitches is a mechanism involving the rearrangement of vortices in neutron star interior. Also from the size of its frequency jump, we inferred that the crust of this pulsar is cooler than radio pulsar of similar age like Crab pulsar.

\subsection{Glitch Activities in Young Pulsars}

Very young pulsars have shown high glitch rate. In the context of [13] work, the glitch activity of pulsars correlates well with the spin-down rate $\dot{v}$. This is as expected if one considers the vortex creep model of pulsar glitches. This is because for a faster spin-down the angular velocity lag between the crustal superfluid and the rest of the star would increase faster, being able to reach a critical value in a shorter time. However, the Crab pulsar, which has the largest $\dot{V}$ among the glitching pulsars, does not exhibit large size glitches and its glitch spin-up rate is considerably smaller than that of X-ray PSR J0537-6910, which has a similar $\dot{v}$.

Also in line with [17] in differentiating the Crab and Vela pulsars glitch activity, the difference in glitch activity of PSR J0537-6910 and the Crab pulsar could be related to their ages. Although the characteristic age is not an accurate age estimator, the age of the respective supernova remnants confirm that the Crab pulsar (1Kyr) is younger than X-ray PSR J0537-6910 (5Kyr) and the Vela pulsar is $11 \mathrm{Kyr}$ $[18 ; 19 ; 20]$. In general, Crab-like pulsars, of characteristic age less than $5 \mathrm{Kyr}$, undergo small or medium sized glitches $\left(\Delta v<10^{-6} \mathrm{~Hz}\right)$ except for AXPs, and shows glitch activity lower than older pulsars, like the Vela pulsar. Perhaps higher temperatures in younger pulsars prevent the glitch mechanism from working as efficiently as it does for Vela-like pulsars just as suggested by [3] and [21]. In terms of vortex unpinning, under higher temperatures, thermal fluctuations could compete effectively against pinning forces, there-by impeding the formation of large pinning zones. Moreover, [17] points out that through quakes the Crab pulsar is still creating vortex depletion zones; which means that the time the Crab pulsar is as old as Vela, it will be producing large size glitches just like Vela. Also in line with [17], [22] proposed that in general young pulsars are just creating their first surface cracks, which generate vortex depletion and trap zones, which will later be able to produce the large glitch activity seen in Vela-like pulsars.

\subsection{Conclusion and Recommendations}

Pulsar glitches have been and the only promising way to probe neutron star interior for now. The number of glitching pulsar is too small compared to the known neutron star population. Generalizing the outcome of recent studies and even from other source may be deceiving. Out of 102 pulsars studied, only six have significant number of glitches that for statistical analysis. This number represents less than $0.003 \%$ of the neutron stars population. In addition, the number of glitches from other manifestations of neutron star, other than radio pulsars, is small to strongly generalize the outcome of their results with regard to a particular class.

Moreover, a well-matured neutron star is expected to consist of several superfluid components. This is the main idea behind most glitch model. The analysis of glitch parameters shows that most glitches have a magnitude $(\Delta v / v)$ of between $10^{-8}-10^{-6}$. Changes in frequency derivatives $(\Delta \dot{v} / \dot{v})$ following glitches are supposed be instrument for estimating the amount of superfluid involved in glitch. Due to the errors associated with its measurement, it becomes so difficult to quantify the amount of superfluid involved in a glitch. Moreover, without knowing this it becomes very difficult to say the exact mechanism producing glitch in various manifestations of neutron stars. For now mechanisms involving the rearrangement of vortices still, take the upper hand.

Meanwhile, the glitch observed in millisecond pulsar PSR B1821-24 on MJD 51980 is strong evidence that glitching phenomenon is one of the characteristics features of all neutron stars during their lifetime and support the idea that millisecond pulsars are recycled pulsars.

Finally, we recommend that there should be an improvement in estimation of pulsars age, as this will actually lead to proper understanding of glitch trigger mechanism. A better way for measuring $\Delta \dot{v} / \dot{v}$, and a way to measure the variation of surface magnetic field of neutron stars during glitches should be devised. In addition, a search for another form of angular momentum reservoir should commence in order to test existing theories with incoming ones.

\section{References}

[1] Maran, S.P. 1992, The Astronomy and Astrophysics Encyclopedia, Van Nostrand Reinhold, New York

[2] Gold, T., Rotating Neutron Stars as the Origin of the Pulsating Radio Sources. Nature, 1968, 218, 416

[3] McKenna, J. Lyne, A. G. PSR1737 - 30 and period discontinuities in young pulsars. Nature, 1990, 343, 349

[4] Baym, G., Pethick, C., Pines, D., Ruderman, M., Spin up in neutron stars: The future of the Vela pulsar. Nature, 1969, 224, 872

[5] Wang, N., Manchester, R.N., Pace, R.T., Glitches in southern pulsars. MINRAS, 2000, 317, 843

[6] Espinoza, C.M., Lyne, A.G., Stappers, B.W, Kramer, M., A study of 315 glitches in the rotation of 102 pulsars 2011, MNRAS, 414, 1679. 
[7] Ruderman, M., Neutron Star quakes and pulsar periods. 1969 , Nature 223, 597

[8] Ruderman, M., Neutron Star crustal plate tectonics. I Magnetic dipole evolution in millisecond pulsars and low-mass X-ray binaries. ApJ, 1991, 366, 261

[9] Ruderman, M., Zhu T., Chen K., Neutron Star Magnetic Field Evolution, Crust Movement, and Glitches. ApJ, 1998, 492, 267

[10] Anderson, P.W., Itoh, N., Pulsar glitches and restlessness as a hard superfluidity phenomenon. Nature, 1975, 256, 25

[11] Ruderman, M., Crust-breaking by neutron superfluids and the VELA pulsar glitches. ApJ, 1976, 203, 213

[12] Alpar, M. A., Anderson, P.W., Pines, D., Shaham, J., Giant glitches and pinned vorticity in the VELA and other pulsars. ApJ, 1981, 249, 129

[13] Urama, J. O., Okeke, P. N. Vela-size glitch rates in youthful pulsars. MNRAS, 1999, 310, 313

[14] Lyne, A. G., Hobbs, G., Kramar, M., Strirs I., Stappers, B., Switched Magnetospheric Regulation of Pulsar Spin-Down. Science, 2010, 329, 408

[15] Dib, R., Kaspi, V.M., Grviil, F.P., Glitches in Anomalous X-ray Pulsars. , ApJ, 2008 673, 1044
[16] Link, B., K., Epstein, R., I., Thermally Driven Neutron Star Glitches. ApJ, 1996, 457, 844

[17] Alpar, M. A., Chau, H. F., Cheng, K. S., Pines, D., Postglitch Relaxation of the Crab Pulsar after Its First Four Major Glitches: The Combined Effects of Crust Cracking, Formation of Vortex Depletion Region and Vortex Creep. 1996, ApJ, 459, 706

[18] Comella, J. M., Craft H. D., Lovelace, R. V. E., Sutton, J. M., Tyler G. L., Crab Nebula Pulsar NP 0532. Nature, 1969, 221, 453

[19] Aschenbach, B., Egger R., Trumper J., Discovery of explosion fragments outside the Vela supernova remnant shock-wave boundary. Nature, 1995, 373, 587

[20] Marshall, F. E., Gotthelf, E. V., Zhang W., Middleditch, J., Wang Q. D., Discovery of an Ultrafast X-Ray Pulsar in the Supernova Remnant N157B. ApJ, 1998, 499, L179

[21] Link, B., Epstein, R.I., Lattimer, J.M. Pulsar Constraints on Neutron Star Structure and Equation of State. Phys. Rev Lett., $1999,83,3362$

[22] Middleditch, J., Marshall, F. E.,Wang, Q. D., Gotthelf, E. V., Zhang W., Predicting the Starquakes in PSR J0537-6910. 2006, ApJ, 652, 153. 